\title{
The research of Media streaming technology based on RTP protocol
}

\author{
Peng Cheng ${ }^{1, a}$, Hui Qu ${ }^{2, b}$ \\ ${ }^{1}$ Training Department, Engineering College of CAPF, Xi'an 710086, China \\ ${ }^{2}$ Faculty of Science, Engineering College of CAPF, Xi'an 710086, China \\ acpskull@163.com, bqh3798150@hotmail.com
}

Keywords: Media streaming technology; RTP-based; Multimedia technology.

\begin{abstract}
Media streaming technology is one of the hot spots in recent research of networking domain. With the development of mobile communication, this technology is applied more and more to the mobile devices. The research of the paper is to research an RTP-based streaming media real-time transmission system design and implementation based on the basic theory of multimedia technology and networks protocol.
\end{abstract}

\section{Introduction}

Media streaming on mobile device also follows the three common requirements, which are being real-time, flatness and synchronization. Designing a high-power transport protocol plays a crucial role in carrying out the object. Real-time Transport Protocol, called RTP for short, is the mainstream during all kinds of transport protocols. It makes use of UDP to transfer media data, because UDP helps to improve the real-time ability. At the same time, it collects the state information of network, so the upper layer applications can control the transport process accordingly. That is why RTP is the first choice in media streaming transportation.

Streaming is a new concept on the Internet in recent years, its definition is widely used, mainly is a term referring to the transmission of multimedia data through network technology. Streaming media includes two kinds of meaning in broad and narrow sense: The term of streaming media in the broadest sense refers to the technology, methods and protocols form stable and continuous flow of transmission, namely the streaming media technology; In a narrow sense, streaming media is compared with the traditional way of download - playback, refers to a kind of get audio and video on the Internet and other new methods of multimedia data, it can support the real-time transmission of multimedia data stream and real-time playback. By using streaming media technology, the server can send stable and continuous flow of multimedia data to the client, the client received data playback at a steady rate, played back rather than after a full download data.

Note that RTP itself does not provide any mechanism to ensure timely delivery or provide other quality-of-service guarantees, but relies on lower-layer services to do so. It does not guarantee delivery or prevent out-of-order delivery, nor does it assume that the underlying network is reliable and delivers packets in sequence. The sequence numbers included in RTP allow the receiver to reconstruct the sender's packet sequence, but sequence numbers might also be used to determine the proper location of a packet, for example in video decoding, without necessarily decoding packets in sequence. While RTP is primarily designed to satisfy the needs of multi-participant multimedia conferences, it is not limited to that particular application. Storage of continuous data, interactive distributed simulation, active badge, and control and measurement applications may also find RTP applicable. This document defines RTP, consisting of two closely-linked parts: (1)the real-time transport protocol (RTP), to carry data that has real-time properties.(2)the RTP control protocol (RTCP), to monitor the quality of service and to convey.(3)information about the participants in an on-going session. The latter aspect of RTCP may be sufficient for "loosely controlled" sessions, i.e., where there is no explicit membership control and set-up, but it is not necessarily intended to support all of an application's control communication requirements. This functionality may be fully or partially subsumed by a separate session control protocol, 


\section{Achievement of Control Mechanism}

Round trip time. The interval of transmitting receiving terminal feedback is based on round trip time (RTT), which is measured by time stamp method. The first RTT calculation directly uses 2 times RTT sample, of which, RTT $_{\text {sample }}=$ Tract-Tlsr-Tdlsr and is the actual RTT sample value, Trcv is the time of receiving RR package, Tlsr is the time of transmitting SR package last time and Tdlsr is the delay after transmitting SR package last time. The sender can carry out package's RTT estimation according to formula (9)based on feedback packet of receiving terminal:

$R T T=\alpha \times$ RTT $_{\text {last }}+(1-\alpha) \times$ RTT $_{\text {sample }}$

Here, RTT last is the round-trip time of package last time, RTT is the round trip time of estimated package, dis weight value and plays smooth role to enhance the response of algorithm to delay, the paper selects 0.87 .

The RTP packet transmission process. RTP, using Mixer (Mixer), and the translator (translator), completes real-time data transmission. Mixer receives big RTP data blocks from one or more of the sender, and put them into a new RTP packet to continue transmit. This combination data blocks will have a new SSRC logo. Especially the sender with a new identity as a special source added to the RTP data block. Because data blocks from different especially the sender cannot synchronous (they can be the different paths through the network), so mixer changed the temporary structure of the media stream. Unlike mixer, the translator only changed data block content, but not the media flow together. The translator only operates on single media stream. (Encoding conversion or protocol translation). A typical RTP packet transmission process is as shown in fig 1:

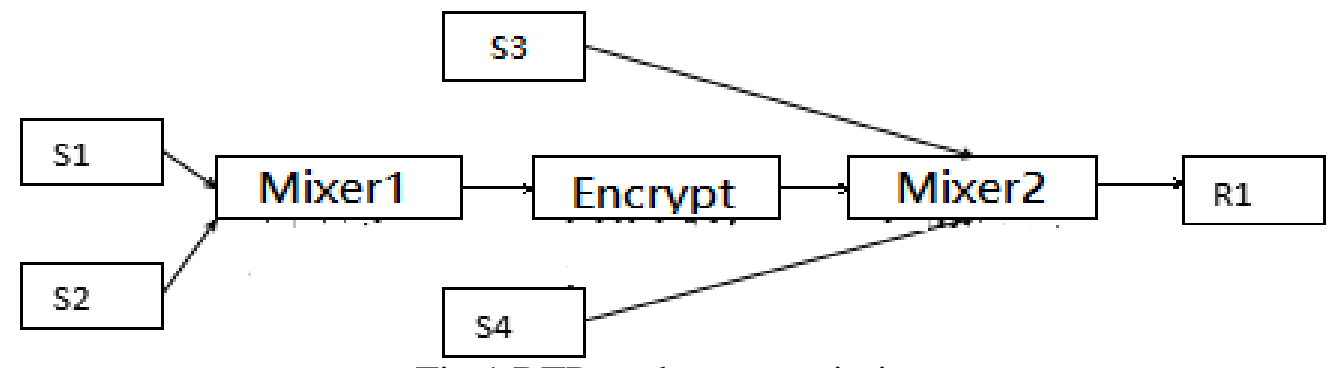

Fig 1 RTP packet transmission

Timeout clock value. The calculation of acknowledgment timeout clock value is mainly responsible by clock control module. The timeout clock value Timeout will be calculated by formula (2) under the situation of no timeout.

$$
\begin{aligned}
& \text { Timeout }=R T T+4 * D \\
& D=(1-\beta) D+\beta \cdot|R T T-M|
\end{aligned}
$$

In the formula, $\alpha$ and $\beta$ are constant, generally, $\alpha=1 / 8, \beta=1 / 4$, $M$ is the round trip time without timeout during recently measured transmission, D is offset, used to make RTT variation be smooth, at the same time, specify the minimum value of Timeout is 1 second to avoid unnecessary timeout.

Transmit control module. The sender adjusts transmission rate through window control mechanism to ensure the quality of streaming media business. The coder will transmit data to transmission control module when the sender needs to transmit data frame. The module decides whether to transmit data according to the relationship between pipe, the data frame number of sender having transmitted but not received acknowledgment at present, and current window size, namely, the maximum continuously transmitted frame number cwnd.

If pipe $<$ cwnd, a data frame can be transmitted and set pipe=pipe +1 ;

If pipe $\geq$ cwnd, this data frame cannot be transmitted and will be abandoned or cached for some time according to certain rules and then transmit, depending on the requirements of specific business.

Every time when transmitting a data frame, will make timeout clock restart to timing if it fails to start, besides, if has started, the timeout clock will not need to restart timing. 
Transmission control mechanism. The window module controls window according to feedback situations to adjust transmission rate, which is the core of achieving congestion control. The window adjustment algorithm is as follows:

It will be thinked that frame loss occurs if exceeding timeout clock value but not receiving acknowledgment information, what shows that, network congestion caused package loss. At this time, set pipe $=0$, the window adjustment threshold ssthreshold $=3 / 4 *$ cwnd and $\mathrm{cwnd}=\mathrm{cwnd} / 2$ to steadily reduce transmission rate or quickly into congestion avoidance state.

If the sender receives acknowledgment frame before the acknowledgment timeout clock, the total number $r$ of actually received and lost frame will be calculate according to the last data frame number feedback by receiving terminal. The algorithm code will be shown as follows:

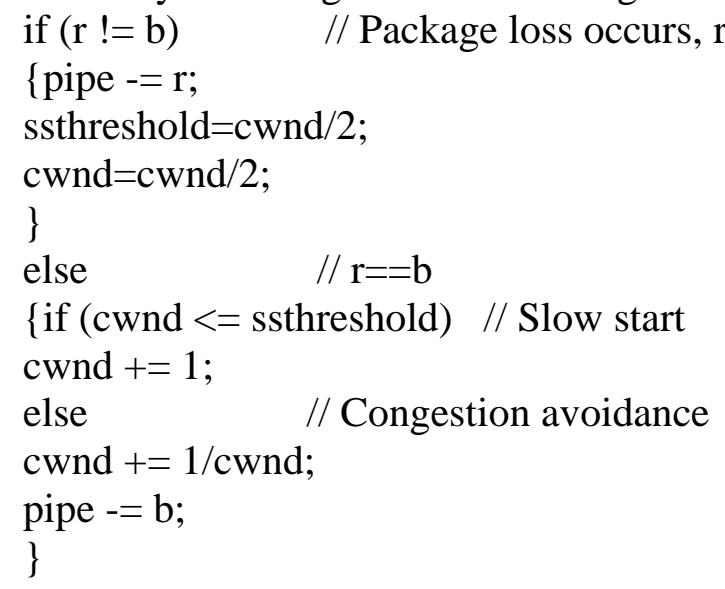

In order to test bandwidth utilization and congestion control ability, we set two software terminals to mutually transmit video streaming with format of H261CIF as well as the highest rate of 400Kbps, and carry out comparison test with RTP/RTCP algorithm. During the experiment, we use interference program transmit package simultaneously on the test link by a certain average bit rate. The variation of transmission rate of two algorithms under interference bit stream with is different bit rate. Of which, the horizontal axis is the time axis (s) and vertical axis is transmission rate (Kbps). During the video transmission, the two algorithms' terminals will adjust average rate of interference program for one time every 15 minutes. The adjustment process of interference program bit rate is: 64Kbps-128Kbps- 256Kbps- 128Kbps-64Kbps.

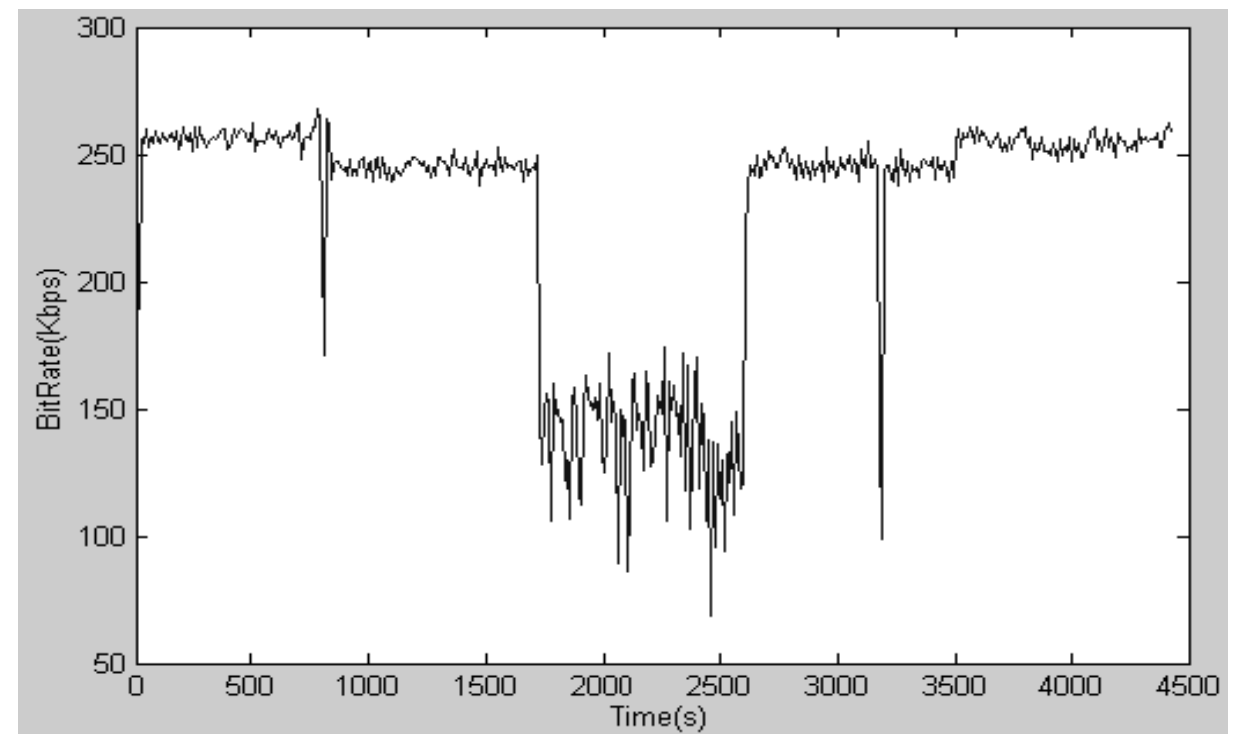

Fig. 2: Variation Process of TCP-like Algorithm Transmission Rate

The test results show that, the transmission rate can be adjusted quickly to available bandwidth and the rate is stable and bandwidth can be fully used in a longer period based on the proposed algorithm. However, the traditional RTP/RTCP algorithm's vibration is larger. Under the situation of network 
bandwidth fluctuates, the TCP-like algorithm can respond quickly. For example, when the available bandwidth reduces, the TCP-like algorithm can reduce transmission rate within about 20-40 seconds, the process of reducing bit rate is relatively smooth and the variation curve is step wise. However, when the available bandwidth greatly reduces 500-1000s, as shown in Figure 2, the variation of bit rate is faster and the algorithm still needs further improvement on this.

\section{Conclusion}

As the multimedia data is becoming more and more important on the Internet, which need real-time transmission of audio and video ,other multimedia data situation will be used widely, such as IP telephony, video on demand, online meeting. RTP is a protocol used for real-time streaming media transmission on the Internet, now it has been widely used in various occasions.

\section{Reference}

[1] Li Guodong,Jin Pengfei, A dynamically adjusted congestion control algorithm for TCP, Journal of Information and Computational Science, Vol.9, No.16, 2012, 4691-4697.

[2] Sathiaseelan Arjuna, Fairhurst Gorry, TCP-Friendly Rate Control (TFRC)for bursty media flows, Computer Communications, Vol.34, No.15, 2011, 1836-1847

[3]Attiya, Gamal, New Strategy for Congestion Control based on Dynamic Adjustment ofCongestion Window, International Journal of Computer Science Issues, Vol.9, No.2, 2012, 368-377.

[4] XIAO Fu,WANG Ruchuan,SUN Lijuan, Wireless network based TCP-friendly congestion control mechanism, Computer Science, Vol.37, No.7, 2010, 50-53(in Chinese) 